\title{
Herrschaft kraft Nichtwissen?
}

\section{Politische und rechtliche Folgeprobleme der Regulierung neuer Risiken}

\author{
Von Boris Holzer und Stefan May
}

Zusammenfassung: Die Regulierung technischer und anderer »neuer« Risiken stellt den vorläufigen Endpunkt einer Entwicklung des staatlichen Umgangs mit Gefährdungen dar, die von der klassischen Gefahrenabwehr (Rechtsstaat) über die Vorsorge für individuelle Notlagen (Sozialstaat) zur Bewältigung kollektiver Gefährdungslagen (Präventionsstaat) führt. In diesem Prozess haben sich nicht nur die zentralen Ziele staatlichen Handelns von der Rechtssicherheit zur sozialen Wohlfahrt und dann zur Risikovorsorge verändert, sondern auch seine Instrumente. Die neuere Staatstheorie vertritt die These, dass gegenüber dem klassischen Zwangsapparat der Macht und der wohlfahrtsstaatlichen Steuerung durch Geld nun »Wissen« an Bedeutung gewonnen hat. Wir modifizieren dieses Argument dahingehend, daß nicht überlegenes oder auszuhandelndes Wissen, sondern gerade der kompetente Umgang mit Nichtwissen und Ungewissheit zu einer zentralen Ressource politischer Entscheidungen wird. Nicht die Eliminierung von Unsicherheit durch überlegenes Wissen, sondern vielmehr die ständige Absorption von Unsicherheit erlaubt es staatlichen und nichtstaatlichen Akteuren, sich als Sachwalter politischer Entscheidungen zu inszenieren. Dies wirft ein neues Licht auf das klassische Modell einer »Herrschaft kraft Wissen«, die Weber als Grundzug der vom Fachbeamtentum beseelten modernen Bürokratie erschien.

\section{Einleitung}

Regulative Politik gehört zum Kernbereich staatlichen Handelns. Darüber herrscht Übereinstimmung in der politischen Theorie, angefangen von Hobbes und Locke bis zu den zeitgenössischen liberalen, realistischen und marxistischen Staatstheorien. Sowohl der Schutz individuellen Eigentums gegen den Zugriff anderer als auch die Beschränkung radioaktiver Emissionen greifen auf die Mittel des staatlichen Gewaltmonopols zurück. Es ist aber offensichtlich, dass sich dabei gänzlich verschiedene Probleme für die Wahl und Rechtfertigung der eingesetzten Mittel ergeben. Die Form regulativer Politik hat sich deshalb mit der technologischen und wissenschaftlichen Entwicklung wesentlich verändert. Da neue Elemente sich jedoch mit alten mischen und überlagern, laufen wir sowohl bei einer zu breit angelegten als auch bei einer Einzelaspekte herausgreifenden Analyse moderner »Staatsaufgaben« Gefahr, eine »Revolution unter dem Deckmantel der Normalität« (Beck) zu übersehen.

Ausgangspunkt unserer Überlegungen ist die Feststellung, dass die Veränderung (und zunehmende Bedeutung) regulativer Politik sich als Ausdruck einer bestimmten Entwicklungslinie des staatlichen Umgangs mit Gefährdungen interpretieren lässt. Diese führt von der klassischen Gefahrenabwehr (Rechtsstaat) über die Vorsorge für individuelle Notlagen (Sozialstaat) zur »Bewältigung kollektiver Gefährdungslagen« (Präventionsstaat) (Habermas 1992, S. 524f.). Im Zuge der entsprechenden Transformation haben sich nicht nur die zentralen Ziele staatlichen Handelns geändert - von der Rechtssicherheit zur sozialen Wohlfahrt und dann zur Prävention -, sondern auch die Medien regulativer Politik. Nicht mehr allein Macht und Geld flankieren die Umsetzung politischer Entscheidungen; diese müssen sich vermehrt um die Rezeption und Erzeugung von »Wissen« bemühen, um in einer durch neue technische Risiken geprägten Umwelt anpassungsfähig zu bleiben (1.). Dies wurde als Anzeichen für eine schleichende »Technisierung « des Rechts und einer folgerichtigen Abdankung der Politik interpretiert (2.). Wir modifizieren dieses Argument dahingehend, dass in den Aushandlungsprozessen zwischen Verwaltung und Regulierten nicht nur Wissen, sondern gerade Nichtwissen zu einer zentralen Ressource wird (3.). Dass professionelle Exper- 
ten in besonderer Weise legitimiert erscheinen, die resultierende Ungewissheit zu absorbieren, darf aber nicht so interpretiert werden, dass diese die institutionelle Politik schlichtweg ersetzten (4.). Nicht etwa die Eliminierung von Unsicherheit durch überlegenes Wissen, sondern vielmehr die ständige Absorption von Unsicherheit durch epistemische Autorität kennzeichnet das Verhältnis zwischen staatlicher Regulierung und nichtstaatlichen Akteuren in jenen Bereichen, in denen »neue Risiken« das Bild prägen.

\section{Von der Gefahrenabwehr zur Risikoprävention}

In der Entwicklungsgeschichte westlicher Staaten beobachten wir eine deutliche Akzentverschiebung von der Abwehr von Gefahren im liberalen Rechtsstaat zur Prävention von Risiken im Vorsorgestaat. Im Selbstverständnis des Rechtsstaats dominiert die Idee der Gefahrenabwehr: Anlass staatlicher Intervention sind vor allem Störungen einer gegebenen Ordnung, die über das Polizeirecht abgewickelt werden; es gilt die »Zuordnungs-Trias von Gefahr, Verursachungszusammenhang und ordnungspflichtigem Störer « (Wolf 1988, S. 169). Gerechtfertigt sind danach prinzipiell nur punktuelle Eingriffe vor dem Hintergrund selbstorganisierter Stabilität, die Verwaltung operiert und interveniert auf der Basis von Konditionalprogrammen. Demgegenüber bedeutet der Sozialstaat eine Materialisierung des Rechts: Nicht allein die Bewahrung einer sozialen Ordnung, sondern ihre Veränderung und Gestaltung stehen im Vordergrund. Die Entscheidungskoordinaten des Sozialstaates erscheinen im Vergleich zum rechtsstaatlichen Modell punktueller und stabilisierender Interventionen als zukunftsgerichtet, flächendeckend und gruppenbezogen; dies geht einher mit einer zunehmenden Relevanz von Finalprogrammen in der Verwaltung, die dieser ein vermehrt zielorientiertes statt nur anlassbezogenes Entscheidungsverhalten zumuten (Grimm 1991). Ist der Sozialstaat die politische Antwort auf die mit der industriellen Klassengesellschaft entstandene soziale Frage, so findet in jüngster Zeit eine »Neubelebung der Staatsdiskussion « (Fürst 1987) vor dem Hintergrund eines gesellschaftlichen Wandels statt, der zu einer Ausdehnung der Staatsaufgaben im spätmodernen Präventionsstaat führt. Unter dem Eindruck »neuer Risiken« werden Schwierigkeiten des adaptierten Polizeirechts deutlich, das offenbar keine ausreichende Handhabe bietet, um technische Risiken auf der Grundlage hergebrachter Vorstellungen von einer »objektiven Gefährdungslage« unter Kontrolle zu bringen. Die neuere Risikoregulierung orientiert sich deshalb immer weniger am Modell polizeilicher Intervention und immer mehr an dem wissenschaftlich informierter Vorsorge (vgl. Beck/May 2001)

\subsection{Folgeprobleme für moderne Staatlichkeit}

Gab der liberale Staat Rahmenregelungen vor und sorgte der Sozialstaat für sozialen Ausgleich, so muss der Staat nunmehr in ungekanntem Ausmaß steuernd in die Gesellschaft eingreifen: Einen eingeschränkten Katalog von Staatsaufgaben gibt es nicht mehr, ${ }^{1}$ prinzipiell jeder Sachverhalt ist der gesellschaftlichen (Re-)Konstruktion als Risiko zugänglich und damit politisierbar (Luhmann 1991, S. 171ff.). Diesem umfassenden Bedürfnis nach Sicherheit kann der Staat weder repressiv noch restitutiv, sondern nur präventiv begegnen. Sicherheit soll der Staat zum einen nicht mehr in Form von Rechtssicherheit durch nachträgliche Schadensregulierung (Strafe/Schadensersatz) bewirken, sondern in Form von Rechtsgütersicherheit (Denninger 1988), indem er jegliche Rechtsgutgefährdung schon im Ansatz aufspürt und vorbeugend verhindert.

1) Mangels materieller Abgrenzungskriterien ist deshalb vom »System der offenen Staatsaufgaben« die Rede, in welchem allein der Gesetzgeber darüber entscheidet, welche Aufgaben und gegebenenfalls in welcher Form er diese wahrnehmen will: vgl. dazu den Zwischenbericht des Sachverständigenrats der Bundesregierung »Schlanker Staat« vom Januar 1996, S. 6. 
Die weitgehende, mit dem modernen Präventionsstaat verbundene Eingriffsintensität ist erst ersichtlich, wenn man sich den Gegenstandsbereich präventiver Staatstätigkeit vor $\mathrm{Au}-$ gen führt. Einerseits werden die natürlichen Lebensgrundlagen potentiell von jedermann zu jeder Zeit gefährdet. Umweltverschmutzung ist die - häufig nichtintendierte - Nebenfolge nicht nur erlaubten, sondern prinzipiell erwünschten Verhaltens. Gleiches gilt für die Produktion anderer Risiken. Objekt präventiver Staatstätigkeit ist damit potentiell jedes mögliche Verhalten, und jedermann ist potentiell verdächtig. Andererseits ist im Bereich der Hochtechnologie (z.B. Atom- und Biotechnologie) sowie beim Schutz der Funktionsfähigkeit bestimmter Systeme (z.B. Datennetze) zwar der Kreis potentiell schädlicher Handlungen eingrenzbar, aber die Schadenshöhe erreicht ein inakzeptables Ausmaß, so dass die staatliche Prävention so weit ins Vorfeld potentiell schädigender Handlungen verlegt wird, dass ebenfalls ganze Personengruppen und ganze Handlungsbereiche ins Blickfeld der Prävention geraten. Zudem versagen Repression und Restitution als Mittel staatlicher Politik immer dort, wo entweder Täter und Kausalitäten nicht feststellbar sind oder aber die Schäden ein Ausmaß erreichen, das sich einer monetären Kompensation entzieht. Wo die Institution des Schadensersatzes versagt, muss der Staat schon den Eintritt eines Schadens präventiv verhindern. In der Folge verändern sich die Gegenstandsbereiche der Vorsorge, und es kommt zu einer Vorverlagerung der Staatstätigkeit in ehemals »staatsfreie« Zonen. Das rechtsstaatliche Problem der Begrenzung des Staates gewinnt daher mit der Prävention neue Aktualität (vgl. Backes 1986, S. 340ff.; Hesse 1994).

Beobachter führen dies nicht nur auf das Auftauchen neuer Risiken, sondern auf einen nach wie vor unklar konturierten gesellschaftlichen Wandlungsprozess zurück. Die konkurrierenden Beschreibungen der Gesellschaft als Wissens-, Informations-, oder Risikogesellschaft und die korrespondierenden Konzepte vom gärtnernden, sorgenden oder präzeptoralen Staat, vom Sicherheits-, Präventions- und Schutzstaat ${ }^{2}$ fokussieren Teilaspekte gegenwärtiger gesellschaftlicher Entwicklungen, die ihre Gemeinsamkeit in einem steigenden Sicherheitsbedürfnis der Gesellschaft finden, das an den Staat als schützende Instanz weitergegeben wird. ${ }^{3}$ Dieses Sicherheitsbedürfnis entsteht vor dem Hintergrund einer zunehmend gesellschaftlich wahrgenommenen und beschriebenen Kontingenz und Ambivalenz (Rorty 1988; Bauman 1992). Unabhängig von der Begriffswahl sollen im folgenden einige wesentliche Merkmale herausgearbeitet werden, die den der neuen Staatsdebatte zugrunde liegenden gesellschaftlichen Wandel charakterisieren. Er kann an drei Aspekten beleuchtet werden, die je für sich mit der gestiegenen Bedeutung von Wissenschaft und Technik für die moderne Gesellschaft in Zusammenhang stehen.

\section{Komplexität und Interdependenz}

Eine Steigerung des Sicherheitsbedürfnisses der Gesellschaft ergibt sich erstens aus der Durchsetzung funktionaler Differenzierung. Die Bildung spezialisierter Funktionssysteme (Wissenschaft, Wirtschaft, Recht, Politik, Erziehung, Gesundheit usw.) in der Gesellschaft führt einerseits zu einem Auseinanderdriften der Gesellschaft, also zu gesteigerten Independenzen, die die gesamtgesellschaftliche Integration zunehmend erschweren (Peters 1993). Gleichzeitig begründet die funktionale Differenzierung aber neue Interdependenzen, insofern die Subsysteme nicht nur intern immer höhere Komplexitätsstrukturen aufbauen, sondern auch die von ihrer Umwelt vorstrukturierte Komplexität voraussetzen und benutzen (Willke

2) Siehe zu den genannten Begriffen Stehr (1994), Steinbicker (2001), Beck (1986; 1988) sowie de Swaan (1993), Ewald (1986), Di Fabio (1993), Hirsch (1980), Denninger (1988) und Grimm (1986, S. 38ff.), Hesse (1993; 1994).

3) Vgl. aus einer umfangreichen Diskussion: Murck (1980), Zapf (1987), Luhmann (1991, Kap.8), Willke (1992, S. 291). 
1992, S. 60). Die Wirtschaft kann nur deshalb immer unwahrscheinlichere Strukturen aufbauen, weil sie ein für sie in seiner Komplexität zwar nicht durchschaubares, für ihre Bedürfnisse aber funktionierendes Rechts-, Wissenschafts-, Gesundheits- oder politisches System voraussetzen kann, und gleiches gilt jeweils umgekehrt. In diesem komplex strukturierten System der funktionalen Differenzierung wird Information zu Macht und Kommunikation zur Überlebensstrategie. Die sich herausbildende Informations- und Kommunikationsgesellschaft wird nicht nur immer sensibler gegenüber jeder Art von Gewalt, sondern insgesamt störanfälliger, soweit sich jedes spezialisierte Teil der Gesellschaft auf das Funktionieren jedes anderen Teils der Gesellschaft verlassen können muss. Die »Funktionsfähigkeit« des Gesamtsystems bzw. einzelner Teilsysteme ist damit zu einem neuen - mitunter verfassungsrechtlich geschützten Rechtsgut geworden, für dessen Sicherung der Staat einzustehen hat.

\section{Entscheidungsabhängigkeit und Politisierbarkeit ehemals »naturaler Grundlagen«}

Dies zeigt sich zweitens exemplarisch im Bereich des Umweltschutzes. Wissenschaft und Technik bilden zwar von jeher die Grundlage der Aufklärung und der Industrialisierung, aber erst im Verlauf des 20. Jahrhunderts werden sie mehr und mehr zur unverzichtbaren Grundlage der Ökonomie, wobei die Grenzen zwischen Wissenschaft und Technik durch einen zunehmenden Handlungsbezug von Forschung verschwimmen (Willke 1992, S. 273). Ab der Mitte des 20. Jahrhunderts werden dann die negativen externen Effekte der technologischen Entwicklung sichtbar, die Gesellschaft gefährdet sich selbst in einem Ausmaß, das die Fortexistenz der Menschheit in Frage stellt. Diese Selbstgefährdung lässt die der menschlichen Kontrolle entzogenen Gefahren zunehmend in den Hintergrund treten, auch wenn sie keineswegs verschwinden. Die durch den wissenschaftlich-technischen Fortschritt erweiterten Handlungsoptionen führen dazu, dass die Natur selbst in dem Maße entzaubert wird, als mehr und mehr ehemals natürliche Gefahren entscheidungsabhängig werden. Frühwarnsysteme verhindern zwar keine Erdbeben, aber sie verwandeln ein unvermeidbares Schicksal in eine zumindest teilweise entscheidungsabhängige Betroffenheit. Die Globalverantwortung des Staates für die wirtschaftliche, kulturelle und soziale Entwicklung erweitert sich dadurch zu einer Globalverantwortung für die Sicherung der natürlichen Lebensgrundlagen - und zwar auch gegenüber zukünftigen Generationen. ${ }^{4}$ Bei der Lösung dieser Aufgabe ist der Staat zunehmend selbst auf Wissenschaft und Technik angewiesen. Denn diese gefährden nicht nur die natürlichen Lebensgrundlagen, sondern die Gefährdungen sind auch nur aufgrund von Wissenschaft und Technik wahrnehmbar und bearbeitbar. Das Schicksal des Staates wird dadurch unmittelbar mit dem Schicksal der modernen Wissenschaft verknüpft (Beck 1986, S. 254ff.; 1988, S. 115ff.).

\section{Individualisierung, Pluralisierung und Kontingenzerfahrung}

Ein zunehmendes Sicherheitsbedürfnis ergibt sich drittens aus individueller Sicht aus der mit dem rapiden gesellschaftlichen Wandel und der Entwicklung von Wissenschaft und Technik einhergehenden Pluralisierung der Lebenswelten und Individualisierung der Lebensformen. Dort, wo alles nur noch entscheidungsabhängig gilt, wird Zukunft als Risiko erfahren (Luhmann 1991, Kap. 2). Bei der Suche nach Entscheidungsmaßstäben hilft dem Individuum aber auch der Blick auf die Wissenschaft nicht mehr weiter. Denn nachdem diese zunächst alle überlieferten Werte und Autoritäten in Frage stellte, ist mit der Durchsetzung von Wissenschaft und Technik als generellem Problembewältigungsmuster die Wissenschaft selbst als objektive Instanz entzaubert worden, was als Zeichen eines Übergangs zur »Postmoderne« gewertet wird (Lyotard 1986). Die Wissenschaftsgesellschaft hat eine »Explosion des

4) Vgl. den durch Gesetz v. 27.10.1994 eingefügten Art. 20 a GG. 
Wissens « bewirkt, wobei sich jeder einzelne im Rahmen der Spezialisierung auf den Punkt zubewegt, wo er »alles von nichts« weiß. Die Zunahme gesellschaftlichen Wissens hat nicht zu mehr Sicherheit, sondern zu mehr Unsicherheit geführt, weil mehr Wissen auch mehr Wissen über das, was man nicht weiß, also mehr Unwissenheit zur Folge hat. ${ }^{5} \mathrm{Mit}$ der $\mathrm{Zu}-$ nahme von Wissen widerspricht sich die Wissenschaft zunehmend selbst. Wo Experten und Gegenexperten über Risiken und Sicherheit streiten, wird die Kontextabhängigkeit allen Wissens offenbar. Die Erkenntnistheorie des radikalen Konstruktivismus (vgl. Luhmann 1994) wird so wie von selbst zum gesellschaftlichen Alltagswissen und Ironie zur Überlebensstrategie. Wem aber die Ironie nicht liegt, der erfährt die Kontingenz der Wahrheit und die Ambivalenz der Werte, die Nichtfeststellbarkeit von Täterschaft, das Versagen der klassischen Zurechnungskategorien von Kausalität und Schuld, die »organisierte Unverantwortlichkeit« (Beck 1988, S. 96ff.) als »neue Unübersichtlichkeit« (Habermas 1985) oder auch einfach als Chaos und gibt sein Bedürfnis nach einfachen Antworten an einen starken Staat weiter, der angesichts des hilflosen Ertragenmüssens systemisch produzierter Risiken Abhilfe schaffen soll.

\subsection{Rechtsformenwandel}

Rechtsintern artikuliert sich dieser Gewissheitsverlust vor allem im Zerfall dogmatischer Leitlinien und methodischer Unsicherheit. Dieser Rechtsformenwandel ist von Autoren wie Max Weber, Franz Neumann, Ernst Forsthoff, Franz Wieacker, Niklas Luhmann, ErnstWolfgang Böckenförde oder Stefan Breuer als Rationalitätsverlust modernen Rechts diagnostiziert worden, in dessen Folge es zu einem dramatischen Anstieg der Unbestimmtheit des Rechts kommt (vgl. Teubner 1989, S. 129). Zwar bleibt die Einheit der Rechtsordnung durch die Rechtswissenschaft äußerlich gewahrt, in seiner Substanz wird das Recht aber nun durch ökonomische, ethische, soziologische und technische Subventionierungen stark heterogenisiert, was sich etwa auch in einer zunehmenden Unschärfe in der Abgrenzung von Gesetzgebung und Rechtsprechung manifestiert (Luhmann 1993, S. 318f.). Es ist aber keinesfalls zwingend, in diesem historischen Rechtsformenwandel ausschließlich einen Rationalitätsverfall zu sehen. Mag das bürgerliche Formalrecht ein kunstvoll konstruiertes System gewesen sein, so ist es doch genauso unbestreitbar, dass dieses System von Anbeginn einen entscheidenden Makel hatte: Es setzte nämlich eine Umwelt voraus, die sich gar nicht und wenn überhaupt - dann nur in vorhersehbaren Bahnen änderte; dagegen reagieren die flexiblen Rechtsformen der sozialstaatlichen Massendemokratie gerade auf den Zerfall der Bedingungen, die dem liberalen Gleichgewichtsdenken zugrunde lagen. Die Rationalität der sozialstaatlichen Rechtsformen lag und liegt genau darin, das Rechtssystem auf immer schnellere Umweltveränderungen einzustellen und durch pluralistische Arrangements auch dort eine Ordnungsbildung zu ermöglichen, wo sich die Rechtsfindung nicht mehr durch bereits feststehende Regeln leiten 1ässt (Willke 1992, S. 175ff.). Es ist also keineswegs zwingend, in der Schwäche eines Gesetzgebers, der nicht mehr einen souveränen Willen positiviert, sondern durch Abkommen und Kompromisse ein neues, heterogenes Recht schafft, ausschließlich einen Rationalitätsverfall zu sehen.

\section{Risikoprävention und Expertenwissen: Die Technisierung von Recht und Politik?}

Unbestritten ist dagegen, dass sich die Akzente von der Abwehr von Gefahren auf die Bewältigung schwer zurechenbarer, möglicherweise irreversibler technischer Risiken verschoben haben. Im Umgang mit den Risiken persönlicher Daseinsvorsorge haben sich bereits die

5) Siehe u.a. Beck (1986, S. 254ff.), Denninger (1992, S. 131ff.). 
Grenzen politischer Steuerung durch Macht gezeigt. Doch auch das für die Ziele des Sozialstaats zentrale Medium Geld scheitert an der Aufgabe, die Sicherheitsbedürfnisse angesichts technischer Risiken zu bedienen. Hier kommt der Ressource »Wissen« zentrale Bedeutung zu. Wenn es sich bei (Risiko-)Wissen um eine Ressource handelt, liegt die Frage nahe, wer über sie disponiert. Genauer ausgedrückt: Da Wissen keine Ressource in dem Sinne darstellt, dass sie dem Zugriff anderer entzogen werden kann, geht es vor allem darum, welchen Gruppen, Professionen oder sonstigen »epistemic communities« die Fähigkeit zugeschrieben wird, ein solches Wissen verwalten, formulieren und in Konflikten repräsentieren zu können.

Es ist offensichtlich, dass die Prävention und Regulierung von neuen Risiken sehr viel stärker als die rechtsstaatliche Abwehr von Gefahren oder die sozialstaatliche Gewährleistung von Inklusion auf Expertenwissen angewiesen ist. Die Erwartung - und für manche: die Befürchtung - liegt nahe, dass die Aufgabe, Vorsorge für eine unsichere Zukunft zu treffen und Risiken abzuwenden, denjenigen zugewiesen wird, die aufgrund ihres professionellen Status einen Zugang zu privilegiertem Risiko-Wissen in Anspruch nehmen können. Das heißt: In Frage kommen jene natur- und ingenieurwissenschaftlichen »Risiko-Experten«, die Fragen der Risikoabschätzung in eine entscheidungsfähige Form bringen können, z.B. durch Messung, Standardisierung und Quantifizierung. Die Problematik dieser Konstellation liegt nun nicht allein darin, dass man den Eindruck haben könnte, hier werde der Bock zum Gärtner gemacht, indem jene technisch-instrumentelle Rationalität, die einen wesentlichen Anteil an der Produktion neuer Risiken hat, nun eben diese zu kontrollieren helfen soll. Eine »Technikkontrolle durch Technik «(Wolf 1987, S. 365ff.) mag durchaus effektiv sein. Doch sie wirft die Frage auf, welche Folgen eine Verlagerung von Normierungsprozessen aus dem Recht in den Kompetenzbereich der Natur- und Ingenieurswissenschaft hat. Was heißt es, wenn nicht mehr der Gesetzgeber, sondern der naturwissenschaftliche Sachverstand entscheidet, welche Risiken nach dem »Stand der Technik« zu akzeptieren sind?

Unzweifelhaft muss die juristische Tatsachenfeststellung dort, wo Gefahren und Schäden der direkten Wahrnehmung entzogen sind, in Abhängigkeit von technischen und theoretischen Registrierungs- und Bewertungsapparaten geraten. Das Recht partizipiert damit an der »Gefährdungsverelendung « in der Risikogesellschaft, in deren Verlauf die Betroffenen in Sachen ihrer eigenen Betroffenheit unzuständig werden (Beck 1986, S. 69f.). Für Recht und Politik ist Wissensabhängigkeit in diesem Sinne nichts Neues. Im »klassischen« Modell sind Tatsachenfeststellung und Normierung bzw. Entscheidung jedoch scharf getrennt. Der Ingenieur ist zwar unverzichtbar zur Bestimmung von Gefährdungen, er legt aber nicht die Standards ihrer Bewertung fest. Im Gegensatz dazu scheint die neue Form der Expertenabhängigkeit den »Primat des Rechts« (Wolf 1987, S. 372), d.h. den Vorrang politisch gesetzter Normierung vor der Normierung durch technische Gremien, in Frage zu stellen. Dies liegt lauf Wolf daran, dass das naturwissenschaftlich-technische Fachwissen zum Einfallstor für eine Transformation der staatlich-polizeilichen Gefahrenabwehr in eine ingenieurwissenschaftliche Risikosteuerung wird. Nicht mehr die »Herrschaft kraft Wissen«, die Weber (1980, S. 129) als »Grundcharakter« der vom Fachbeamtentum beseelten modernen Bürokratie erschien, sondern eine »Herrschaft kraft Ingenieurwissen « kennzeichne demnach die Stellung des regulativen Rechts in der Risikogesellschaft (Wolf 1987, S. 365ff.; 1988, S. 172ff.).

Es könnte allerdings sein, dass in dieser Argumentation die Bedeutung und Effizienz von Wissen zu hoch veranschlagt wird. Um es zu einer herrschaftsrelevanten Ressource zu stilisieren, muss man dem naturwissenschaftlichen Wissen schon ziemlich viel Eindeutigkeit und Homogenität zuschreiben. Dies entspräche den Annahmen klassischer TechnokratieThesen, die damit rechneten, dass der Staat sich in seiner wachsenden Abhängigkeit von den Leistungen der modernen Technik zunehmend mit dieser identifizieren werde - um letztlich die Entscheidungshoheit an sie abzutreten (vgl. Forsthoff 1971). Dies ist aber nur dann denkbar, wenn die ehedem durch politische Entscheidung hergestellte Eindeutigkeit sich relativ 
direkt aus den »Sachgesetzlichkeiten der wissenschaftlich-technischen Zivilisation« (Schelsky 1965, S. 453) ergäbe.

Im Gegensatz dazu erkennt allerdings auch Wolf (1988, S. 173), dass die »stille Technisierung « des Rechts alles andere als einheitlich ist, sondern sich vielmehr in einer unüberschaubaren Normenvielfalt, heterogenen Regelwerken und ständigem Änderungsbedarf niederschlägt. Diese Diagnose ist zunächst auf die Problematik umweltpolitischer Grenzwerte zugeschnitten. Dort wird die Uneindeutigkeit naturwissenschaftlichen Wissens insofern invisibilisiert, als sie zwar im Vorfeld als Streit der Experten beobachtbar sein mag, nach der Entscheidung über Akzeptanzniveaus aber nicht mehr »erinnert « wird. Die frühen Vorbehalte gegen eine Technisierung regulativer Politik richteten sich vor allem gegen diesen umweltpolitischen Dezisionismus. Diese Engführung versperrt aber den Blick dafür, dass sich im Bereich der Umweltpolitik ebenso wie in anderen Feldern ein viel tiefgreifenderer Wandel regulativer Politik vollzogen hat. Dieser betrifft nicht länger nur die sozialen Quellen von Risikowissen und damit die »Urheber« entsprechender Standards, sondern die Form regulativer Politik selbst. Wenn Fortschritt der Wissenschaft nicht mehr Sicherheit, sondern mehr Ungewissheit bedeutet, kann auch der Rückgriff auf Expertenwissen nicht als schlichte Verlagerung der Entscheidungskompetenz verstanden werden.

Wir möchten dies kurz anhand zweier Beispiele aus aktuellen Forschungsprojekten illustrieren. ${ }^{6}$ Das erste betrifft die Rolle humangenetischen Experten- und Risikowissens in der modernen Biomedizin. Hier werden durch neue Technologien, insbesondere durch Methoden der Gendiagnostik, neue Krankheitsrisiken sichtbar, die aber weder behandelbar noch in jedem Fall prognostizierbar sind. Der Arzt kann in diesem Fall zwar über statistische »Risiken« (bzw. genauer: Risikowahrscheinlichkeiten) informieren, häufig aber keine Therapie anbieten, mitunter sogar gar nicht zweifelsfrei feststellen, ob eine Therapie nötig ist oder nicht. Trotz ihrer unbestrittenen professionellen Kompetenz in der Entwicklung und Anwendung gentechnischer Verfahren können Humangenetiker in der Kommunikation mit Patienten deshalb kein überlegenes Rezeptwissen anbieten, sondern müssen ihre professionelle Kompetenz vor allem dadurch beweisen, dass sie die durch das Wissen über mögliche Risiken produzierte Unsicherheit handhabbar machen. Die Humangenetik ist geprägt durch ein Therapiedefizit, d.h. durch die Kluft zwischen immer umfassenderen Diagnosemöglichkeiten und kaum vorhandenen Therapiemöglichkeiten (vgl. Schmidtke 1995). Die dadurch entstehende Lücke kann nun gerade nicht durch den individuellen Arzt oder - per programmatischer Vorgaben - durch seine Profession und deren Expertenautorität allein geschlossen werden, auch wenn es zum klassischen professionellen Autonomieverständnis gehört, dass der Arzt das Definitions- und Deutungsmonopol über Krankheit und Gesundheit des Patienten besitzt. ${ }^{7}$ Dagegen spielt in der humangenetischen Praxis gegenüber den Laien weniger die Gewissheit der Diagnose, als vielmehr die Ungewissheit ihrer Folgen eine Rolle. Der Arzt konstruiert sich nicht mehr als Experte, der aufgrund seines größeren Wissens Handlungsanweisungen für den Patienten begründen kann. Das Deutungsmonopol des Arz-

6) Wir beziehen uns im folgenden auf die Ergebnisse von zwei separaten Forschungsprojekten, die von den Autoren zusammen mit Ulrich Beck innerhalb des Sonderforschungsbereichs »Reflexive Modernisierung « bzw. in Assoziation mit diesem durchgeführt wurden. Es handelt sich zum einen um eine Untersuchung von rechtspolitischen Folgen der Biomedizin (Beck/May, Teilprojekt A7 des SFB 536), zum anderen um eine Studie neuer Instrumente der Risikoregulierung im Finanzbereich (Beck/ Holzer, Forschungsprojekt gefördert durch die World Society Foundation, Zürich). Für ausführlichere Darstellungen der Projekte siehe Beck/May (2001), May/Holzinger (2003) und May (2002; 2004) bzw. Holzer/Beck (2004) und Holzer/Millo (2005).

7) Dieses paternalistische Modell orientiert sich »an einem objektiv bestimmbaren Gesundheitsinteresse des Patienten, über das der Arzt als Experte urteilt und aus dem ärztliche Anordnungen nach Maßgabe des professionellen Standards folgen $\ll$ (Francke 1994, S. 39). 
tes als Ausdruck der professionellen Autonomie wird gerade dadurch fragwürdig, dass mit dem humangenetischen Wissen in vielen Fällen keine eindeutige, medizinisch begründbare Handlungsanweisung für die Patienten verbunden ist. Der Humangenetiker vermittelt im Beratungsgespräch statistische Risikowahrscheinlichkeiten, d.h. er kann beispielsweise keine sichere Aussage über den Gesundheitszustand eines geplanten Kindes machen, sondern er muss die Ungewissheit seiner Prognose kommunizieren. Der Wahrscheinlichkeitscharakter der genetischen Information wird von Professionsmitgliedern geradezu als ihr Spezifikum hervorgehoben: »Risiken und Chancen sind Wahrscheinlichkeiten, die sich zufallsmäßig verwirklichen, ohne dass der Berater dies dirigieren kann, schon gar nicht bei der Zeugung eines Kindes« (Schroeder-Kurth 1994). Das damit verbundene Eingeständnis kognitiver Ungewissheit und normativer Unsicherheit verändert den Typus professioneller Autonomie. Durch die Haftungsrechtsprechung zur »fehlerhaften genetischen Aufklärung « sieht sich die humangenetische Profession dazu gezwungen, die Ungewissheit ihres Wissens in der Expertise offenzulegen. In der Folge ist nicht mehr allein das »Wissen« der Experten, sondern vielmehr ihr kompetenter Umgang mit »Nichtwissen« der Dreh- und Angelpunkt ihrer professionellen Kompetenz. Aus der Perspektive einer regulativen Politik heißt dies jedoch, dass man in der humangenetischen Ärzteschaft schlecht eine technisierte Entscheidungsinstanz für die Bewertung genetischer Risiken sehen kann.

Mehr Wissen über Risiken bzw. statistisch ermittelbare Risikowahrscheinlichkeiten führt auch in anderen Bereichen nicht unbedingt dazu, dass sich technische Expertenautorität an die Stelle politischer und rechtlicher Normierungen setzt. Dies lässt sich an einem zweiten Beispiel zeigen, das eine andere, im Hinblick auf die ambivalente Rolle des Expertenwissens aber vergleichbare Konstellation des Umgangs mit Ungewissheit zeigt: der Regulierung von Finanzrisiken. Gerade weil der Begriff des Risikos in diesem Bereich nicht per se auf zu behebende Missstände oder gar zu vermeidende Handlungsalternativen verweist, ist er instruktiv. Das unternehmerische Risiko von Gewinn oder Verlust ist gleichsam konstitutiv für Wirtschaft. Dazu kommt im Fall der Finanzwirtschaft die Tatsache, dass Ungewissheit in Form von unterschiedlichen Zukunftserwartungen den Handel mit Aktien und Finanzderivaten erst attraktiv macht (Baecker 1988; 1991). Trotzdem stellt sich das Regulationsproblem, dass individuelle Risikostrategien mitunter die Stabilität des Gesamtsystems gefährden können. Dies gilt insbesondere für den Bankenbereich, in dem der Kollaps eines großen Kreditinstituts aufgrund der möglichen Kettenreaktionen zu einer Gefährdung des Finanzsystems werden kann (Eatwell/Taylor 2000). Deshalb müssen Vorkehrungen getroffen werden, das Risiko möglicher Ausfälle zu begrenzen, z.B. indem Banken für vergebene Kredite einen Prozentsatz an Eigenkapital zurücklegen müssen (Gallati 2003). Dies lässt sich entweder über ein relatives starres Regime vom Typus »Grenzwerte« erreichen, das für definierte Klassen von Kreditnehmern das zurückzubehaltende Eigenkapital spezifiziert, oder über ein »kooperatives « Regime, das auf dem sehr viel detaillierteren Risikowissen der Banken über individuelle Kredite basiert. Letzteres Modell ist der Inhalt der demnächst einsetzenden Reform der internationalen Bankenregulierung, die unter dem Titel »Basel II« firmiert. Nicht allein von den Aufsichtsbehörden festgelegte Kategorien, sondern auch die Risikobewertungen und -prognosen der Kreditinstitute selbst, sogenannte »interne Ratings«, können dann zur Bestimmung notwendiger Rücklagen herangezogen werden (BCBS 1999; 2001; 2003). Es ist allerdings nur ein Teil der Wahrheit, wenn man das dadurch entstehende Arrangement als eine kooperative »Wissensproduktion « staatlicher und nichtstaatlicher Akteure beschreibt (Lütz 1999; Strulik 2000). Inhalt der Kooperation ist zunächst einmal ein bestimmter Umgang mit den Unwägbarkeiten moderner Finanzmärkte: ein mathematisch-probabilistisches Risiko-Management. Dabei handelt es sich ganz offensichtlich nicht um den Prototyp eines Expertenwissens, das größere Handlungs- und Erwartungssicherheit garantieren kann. Vielmehr ist bereits ohne die auch innerhalb des mathematischen Risikodiskurses wachsende Skepsis gegenüber den Schätzverfahren klar, dass probabilistische Verfahren den Umgang 
mit einer ungewissen Zukunft sozial legitimieren, aber keinesfalls steuern können. Die zentrale Rolle, die nichtstaatliche Akteure in diesem Bereich staatlicher Regulierung spielen, ist deshalb kein Ausdruck überlegenen Wissens. Es handelt sich lediglich um eine Neujustierung dessen, was im Vorfeld der Risikoregulierung festgelegt wird. Keineswegs bestimmen in Zukunft einzelne Kreditinstitute oder die internationale Gemeinschaft der Risikoexperten, welche Modelle oder gar welche Rücklagen zu halten sind. Ihnen wird allein die Entwicklung plausibler Risikomodelle überantwortet, die dann - unter regulativen, nicht wirtschaftlichen Gesichtspunkten - von den Aufsichtsbehörden kontrolliert und abgenommen werden.

Diese beiden Beispiele, die hier nur kurz skizziert werden konnten, sind natürlich kein Beleg dafür, dass es die von Wolf und anderen behaupteten Technisierungstendenzen generell nicht gäbe. Sie verdeutlichen aber, dass gerade dort, wo sich relativ avancierte Probleme der Risikoabschätzung und Ungewissheitsbewältigung stellen, keine »feindliche Übernahme« des regulativen Prozesses zu erwarten ist (oder gar des auf technische Risiken bezogenen politisch-rechtlichen Herrschaftsapparats insgesamt). Sofern es »neue Risiken« gibt, sind diese unzweifelhaft dadurch gekennzeichnet, dass auch diejenigen professionellen und wissenschaftlichen Experten, die einen privilegierten Zugang zur Definition dieser Risiken haben, Ungewissheit nicht mehr durch Sicherheit ersetzen können. Das Versprechen, dort wo Risiko ist, Sicherheit zu schaffen, ist in der Regel weder gesellschaftlich noch innerhalb der betroffenen Gruppen legitimierbar. Es ist deshalb sehr viel wahrscheinlicher, dass ein nichteliminativer Umgang mit Ungewissheit überwiegt und zu einer Neujustierung des Verhältnisses von Recht, Politik und Wissen führt.

\section{Ungewissheit als Problem und Lösung: Recht und Politik des Nichtwissens}

Ungewissheit kann je nach Perspektive ein Problem oder eine Chance für die Erhöhung der Effizienz institutioneller Problemlösungen sein (vgl. Beck 1996; Luhmann 1992; Wehling 2001). Zwar wird die Umstellung von sicherem auf unsicheres Wissen gemeinhin als problematisch in dem Sinne verstanden, dass Routinen und Hintergrundannahmen angepasst werden müssen. Dies schließt es aber keineswegs aus, dass unsicheres Wissen und Nichtwissen in der Folge insofern entproblematisiert werden, als Recht und Politik sich von der Fixierung auf sicheres Wissen lösen. Es stellt sich dann die Frage nach der »Brauchbarkeit von Nichtwissen« (Ravetz 1987), die sich für Recht und Politik in paralleler Weise stellt, aber zu sehr unterschiedlichen Reaktionen Anlass geben kann.

\subsection{Recht und Ungewissheit}

Bei aller Unterschiedlichkeit der jeweiligen Diskussionen ist der gemeinsame Ausgangspunkt die These, dass das Recht der Gegenwartsgesellschaft, das unter der Bedingung von Ungewissheit und Nichtwissen operieren muss, ein »reflexives« Recht sei oder doch zumindest sein sollte. In dieser Diskussion wird zunächst ein heterogenes Feld unterschiedlicher Aspekte wissenschaftlicher Ungewissheit hervorgehoben. Etwa Ungewissheit aufgrund der Produktion hypothetischer, zusammenhangloser und heterogener Detailergebnisse, die Expertendilemmata eröffnet und die Urteilsfindung pluralisiert und politisiert (Beck 1986), oder sicherheitstechnische bzw. kausalitätsbezogene Ungewissheit über die Folgen komplexer Systeme (Perrow 1984). Vereinzelt werden auch Formen erkenntnistheoretischer bzw. wissenschaftstheoretischer Ungewissheit im Nachgang einer selbstkritischen Reflexion der eigenen Rationalitätskriterien diskutiert (Scherzberg 2002, S. 113ff.).

Die grundlegenden Positionen dieser steuerungstheoretisch interessierten rechtssoziologischen Debatten um »Risikorecht «, »reflexives Recht« oder auch »kooperatives Recht« lassen sich wie folgt charakterisieren: In einer ersten Hinsicht geht es darum, ob und in welcher Form dem gegenwärtigen Recht eine Umstellung auf einen neuartigen Modus des »Handelns 
unter Ungewissheit« noch bevorsteht (Wolf 1987), ob das gegenwärtige Recht bereits entsprechenden Veränderungsformen unterliegt (Ladeur 1999) und ob sich in den neuen Regulierungsmodi des Rechts ein gesellschaftlicher Wandel ankündigt (vgl. Ladeur 1995; Gill et al. 1998).

In einer zweiten Hinsicht geht es um die grundsätzliche Modernisierungsbedürftigkeit des Rechts bzw. um die möglichen institutionellen und normativen Folgen einer Modernisierung des Rechts, die aus der möglichen Umstellung des Rechts auf neue Regulierungsformen resultieren. Als mögliche Regulierungsformen des Rechts im Umgang mit Ungewissheit haben sich vier Schwerpunkte herausgebildet, die mit den Stichworten der Flexibilisierung des Rechts, der Temporalisierung des Rechts, der Prozeduralisierung des Rechts und der Partizipationssteigerung oder Reflexivitätssteigerung im Recht charakterisiert werden können (vgl. die Zusammenstellung bei Goeschel 2002). Im Kern reagieren diese neueren Rechtsformen auf die Ungewissheit unbekannter und unerwarteter Handlungsfolgen technisch-wissenschaftlich erzeugter Produkte materieller Art (z.B. FCKW) auf die äußere Natur (z.B. Ozonschild) - m.a.W.: auf modernisierungsbedingte Umweltrisiken und auf deren Lösung mithilfe des Verwaltungsrechts (Dose 2003). Eine Ausweitung dieses Diskurses auf andere modernisierungsbedingte Risiken mit ihren spezifischen Ursachen- und Folgenzuschreibungen beginnt erst allmählich (vgl. hierzu Beck/May 2001; May 2004; 2005a, i.E.). Trotz der Heterogenität der Perspektiven konvergieren diese Diskussionen über das Verhältnis von Recht und Ungewissheit in der Diagnose, dass die Regulierung von Risiken sich nicht bruchlos in das Gefüge modernen Rechts einordnen lässt. So bleibt es letztlich bei der kontrovers diskutierten Frage, zu welchen systematischen Folgen die zunehmende Umstellung des Rechts auf die Regulierung von Ungewissheit und neuer Risiken führt (May 2005).

Selbst eine (teilweise) Umstellung auf die Erwartung des Unerwarteten stellt für ein Funktionssystem, das seinen zentralen Bezugspunkt in der Sicherstellung stabilen und sogar kontrafaktischen Erwartens hat (Luhmann 1972; 1993), eine nicht unwesentliche Irritation dar. Den Vorschlägen einer Prozeduralisierung, Reflexivitätssteigerung oder Temporalisierung des Rechts ist gemeinsam, dass sie die prinzipielle Unbestimmtheit der Zukunft - sowohl was kognitive Erkenntnisse als auch normative Standards betrifft - in die Gegenwart projizieren. Mögliche Enttäuschungen von Erwartungen, die das Recht stets schon antizipiert hat, sollen nicht erst im Falle ihres Eintritts relevant werden, sondern bereits im Vorfeld. Ein auf diese Weise für seine eigenen Folgen sensibilisiertes Recht gerät nicht nur in Probleme, die nötige ständige (Selbst-)Veränderung im Hinblick auf eine unsichere Zukunft mit seinen klassisch auf Vergangenheit orientierten Verfahren und Standards zu vereinbaren (vgl. Hiller 1993). Es wird auch zunehmend zweifelhaft, inwiefern ein reflexives, prozedurales Recht noch als Instrument der Politik, d.h. für die Steuerungszwecke einer im Medium des Recht operierenden politischen Gewalt tauglich ist.

\subsection{Politik und Ungewissheit}

Im modernen Rechtsstaat - der nach wie vor die Grundlage der skizzierten Entwicklungen zum Sozial- und Präventionsstaat bildet - sind Recht und Politik eng miteinander verbunden und wechselseitig aufeinander angewiesen. ${ }^{8}$ Aus der Perspektive des Rechts scheint die kollektive Bindungsfähigkeit von politischen Entscheidungen von deren Rechtmäßigkeit (etwa im Rahmen einer Verfassung) abzuhängen; die Politik wiederum begreift das Recht zum einen als abhängig vom Gewaltmonopol des Staates, zum anderen als komplexes Medium, in das sich politische Entscheidungen (inklusive etwaiger Steuerungsambitionen) einschreiben können. Aus der Perspektive einer Gesellschaftstheorie heißt dies nichts anderes, als dass

8) In Luhmanns (1993, S. 426) Worten handelt es sich um ein »wechselseitig-parasitäres Verhältnis«. 
das eine nicht ohne das andere existieren kann; es handelt sich um »zwei Seiten des Rechtsstaates« (Luhmann 1989). Die skizzierten Probleme, der Ungewissheit einen Platz im Recht zuzuweisen, korrelieren daher mit Veränderungen im politischen System. Dieses muss zum einen damit zurechtkommen, dass bereits vielfach enttäuschte Erwartungen an die Steuerungsfähigkeit des Rechts im Umgang mit neuen Risiken erneut zurechtgestutzt werden; zum anderen kann es sich nicht mehr darauf verlassen, dass das politische Projekt der Herstellung von Sicherheit mit der Entwicklung in anderen gesellschaftlichen Teilbereichen, insbesondere in der Wissenschaft, koordiniert verläuft. Im Gegenteil: Wenn »Fortschritt « in der Wissenschaft nicht mehr als Schritt in Richtung auf Sicherheit und Eindeutigkeit vorausgesetzt werden kann, sondern oft vermehrte Unsicherheit bedeutet, können sich Gewissheitsund Sicherheitsgarantien politischer Entscheidungen nur noch auf Quellen innerhalb der Politik selbst beziehen.

Wenn wir mögliche Quellen autoritativer Entscheidung in der Risikogesellschaft in den Blick nehmen, wird nochmals deutlich, dass diese nicht in den Gewissheitsansprüchen des Ingenieurswissens liegen können. Entsprechend der Formulierung von Friedrich (1958, S. 35) leitet sich Autorität ab aus der zugeschriebenen "potentiality of reasoned elaboration «, also der Fähigkeit, Entscheidungen auf Nachfrage vernünftig untermauern zu können. Dabei geht es um die soziale Zuschreibung und Akzeptanz von Autorität, nicht um ihre sachliche Angemessenheit. Es handelt sich um »Autorität qua Kompetenz«, die sich nicht durch die Richtigkeit spezifischer Informationen auszeichnet, sondern durch das generalisierte Vertrauen in den Mitteilenden; nicht der Inhalt, sondern die Herkunft von Entscheidungen gibt den Ausschlag (vgl. Wrong 1995, S. 52ff.). Wer Unsicherheit im Hinblick auf Tatsachen oder Bewertungsstandards so reduzieren bzw. »absorbieren« kann, dass sich Nachfragen zum Zustandekommen eines Urteils erübrigen, der genießt Autorität und kann diese als Machtressource einsetzen. ${ }^{9}$ Ob Wissenschaftler und Ingenieure Autorität haben, hängt also ebenso wenig direkt davon ab, dass sie Wissen tatsächlich benutzen, wie die legitime Macht des Staates davon, dass er Gewalt einsetzt. In beiden Fällen indiziert das »Austesten« der zugrunde liegenden Ressource vielmehr ein Versagen: So wie der keine ausreichende Macht hat, der Gewalt einsetzen muss, ist es um dessen Autorität schlecht bestellt, der sein Wissen wirklich unter Beweis stellen muss.

Rückt man die oben geschilderten Verweisungen des modernen Risikorechts auf den »Stand der Technik« und das zugrundeliegende Expertenwissen ins rechte Licht, wird aber deutlich, dass diese eher Anzeichen einer relativ geringen Autorität der Wissenschaft sind. Der ubiquitäre Streit der Experten - sowohl im Prozess der Rechtssetzung als auch der -anwendung - spricht in dieser Hinsicht eine deutliche Sprache. Auch die oft vagen und entscheidungsscheuen Beratungskommissionen, die insbesondere im Feld der Biomedizin zu finden sind, weisen nicht auf eine allzu direkt ausgeübte »Herrschaft der scientific community«(Ullrich 1979, S. 311ff.) hin. Daher wäre es viel zu früh, die politische Entscheidung abzuschreiben. Vielmehr kann gerade die Angewiesenheit der Politik auf wissenschaftliche Expertise vor dem Hintergrund unauflösbarer Uneindeutigkeiten dazu führen, dass ihre Entscheidungen umso mehr nachgefragt werden. Die Entfesselung wissenschaftlicher und ethischer Kontroversen über Grenzwerte, Persönlichkeitsrechte und Grenzfragen der Biomedizin diente demnach nicht zuletzt dazu, die Notwendigkeit einer letztlich politischen Entscheidung wirkungsvoll zu inszenieren (Bogner/Menz 2002).

9) Zum Konzept der Unsicherheitsabsorption vgl. March/Simon (1958, S. 165f.). Eine Abgrenzung des Konzepts im Rahmen eines systemtheoretischen Machtbegriffs entwickelt Luhmann (1988; 2000, Kap. 2). 
In der Interaktion zwischen staatlicher politischer Entscheidung und den Risikoexperten der scientific und anderer epistemic communities ergibt sich dadurch eine komplizierte Gemengelage, die mit linearen Formeln à la »Herrschaft kraft Ingenieurwissen« nur unzureichend charakterisiert werden kann. Auch wenn sich auf nationaler wie transnationaler Ebene mittlerweile eine eigene Beratungsindustrie der Wissenschaft herausgebildet hat, die daran arbeitet, der wissenschaftlichen Kontroverse durch Konsensunterstellungen die Brisanz zu nehmen, bleibt die Inanspruchnahme »der « Wissenschaft für eine scheinbare alternativenlose Entscheidungsfindung prekär. Eine klar zurechenbare Einflussnahme ist dort festzustellen, wo es Professionen gelingt, die Sprecherrolle für einen Wissensbereich mehr oder weniger vollständig zu besetzen. Die vertretenen Positionen werden nicht als Entscheidungen, sondern als Wahrheiten oder (im Sinne Schelskys) als Sachgesetzlichkeiten vertreten. Dann liegt der Fall vor, dass die Autorität - im oben genannten Sinne - wissenschaftlichen Wissens die Frage nach Entscheidungsalternativen, ja nach der Notwendigkeit von Entscheidung überhaupt überflüssig macht. Doch eben diese Autorität beruht im Falle der Profession darauf, dass sie Kompetenz im Umgang mit gesellschaftlich relevanten Unsicherheiten beanspruchen kann (Parsons 1939; Stichweh 1994). Der »Test«, ob es sich um ein belastbares Handlungswissen handelt, ist im Routinefall weniger wichtig als die Treuhandfunktion, die anderen die Konfrontation mit Unsicherheit und Nichtwissen erspart.

Nicht die Verfügung über Wissen, sondern vielmehr ein kompetenter Umgang mit Nichtwissen zeichnet deshalb jene Positionen aus, die in der reflexiven Moderne in das Blickfeld einer politischen Soziologie geraten müssten. Wo sicheres Wissen nicht verfügbar ist, wird es entscheidend, wie die aus Nichtwissen resultierende Unsicherheit absorbiert werden kann. Wissen mag - nach wie vor - Macht sein. Doch auch dort, wo es kein Wissen gibt oder zuviel davon (was im Hinblick auf zu bewältigende Kontingenz auf dasselbe hinausläuft), gibt es Macht. Mit der Problematisierung von Wissen und seiner nicht immer wünschenswerten Konsequenzen wird die Kompetenz, mit Nichtwissen umzugehen, zu einer eigenständigen Quelle gesellschaftlicher Macht. Die Frage nach der Kompetenz zur Unsicherheitsabsorption - man könnte in Anlehnung an Marquard (1981) auch sagen: nach der Unsicherheitskompensationskompetenz - lenkt den Blick zwar ebenfalls auf jene Experten, Professionen und epistemic communities, die regelmäßig als Sachwalter steuerungsrelevanten Fachwissens angeführt werden. Sie modifiziert die Analyse allerdings in entscheidender Weise, indem sie die professionelle Selbstbeschreibung überlegenen Wissens zunächst kontingent setzt - statt sie schlicht zu übernehmen und als Erklärung zu akzeptieren.

\section{Entgrenzung von Recht und Politik?}

Je stärker wissenschaftliches Wissen als Antriebskraft für gesellschaftliche Selbstveränderung - etwa im Gegensatz zur Macht - auf die Erforschung des Unbekannten und damit auf die Zukunft abzielt, umso mehr muß die Gesellschaft ständig auf ihre eigenen Veränderungen reagieren und sich dadurch weiter verändern. Dieser Prozess erzeugt aber in seiner Folge einen sich ständig erweiternden Raum von Möglichkeiten zukünftiger Schäden - und Risiken. Risiken, die bestenfalls wieder nur durch neues Wissen erkannt und auch vermieden werden können, oftmals aber gerade in ihren Folgen durch kein aktuelles Wissen vorhergesehen und damit auch nicht behoben werden können (vgl. hierzu Preuß 1996; Beck/May 2001). Das Dilemma der Risikogesellschaft besteht darin, dass die möglicherweise in der Zukunft anfallenden Schäden auf ein regelkonformes (oder zumindest nicht deviantes) Entscheiden in der Gegenwart zurückgehen; nicht Abweichung von Normen, sondern die »Zeitbindung « riskanter Entscheidungen ist das Problem (Luhmann 1990; 1991). Es ist daher nicht überraschend, dass insbesondere vom vergangenheitsorientierten Recht nur begrenzt erwartet werden kann, zukunftsorientierte Risikokonflikte zu entschärfen. Zu den strukturellen Schwierigkeiten des Rechts, mit einer offenen Zukunft umzugehen, tritt zudem der 
Aspekt hinzu, dass die gesellschaftliche Selbstbeobachtung durch die Risikobrille die Stabilitätsgarantien des Rechts unterläuft: »Das Recht kann nicht Sicherheit garantieren, wenn die Gesellschaft selbst ihre Zukunft als entscheidungsabhängiges Risiko begreift« (Luhmann 1993, S. 561).

Doch auch die Rolle der Politik gerät unter dem Eindruck einer durch gegenwärtige Entscheidungen belasteten Zukunft in Bewegung. Denn im Zuge reflexiver Modernisierung wird eine Selbstbeschreibung der nationalstaatlichen Politik obsolet, die dieser nicht nur die Funktion kollektiv bindenden Entscheidens zuweist, sondern sie als zentrale Quelle gesellschaftlicher Selbstveränderung schlechthin ansieht. Die ungeplanten Folgen und Nebenfolgen des naturwissenschaftlichen und technischen Fortschritts machen jedoch deutlich, dass folgenreiche Entscheidungen keineswegs nur in der Politik anfallen. Beck (1986) hat darauf im Zusammenhang mit der Veränderung medizinischer Kompetenzen durch die Gentechnologie aufmerksam gemacht und in den demokratisch nicht legitimierten, aber gesellschaftlich folgenreichen Entscheidungen professioneller Experten eine Form nicht institutionalisierter »Subpolitik « ausgemacht (siehe auch Beck 1993; 1999). Aus dieser Perspektive erscheint »Subpolitisierung « als eine schleichende Aushöhlung staatlicher Institutionen, als ein Rückzug kollektiv bindenden Entscheidens aus der gesellschaftlichen Beobachtung (vgl. auch May/Böschen 2005). ${ }^{10}$ Damit wird allerdings die vielleicht irreführende Vorstellung verbunden, die neuen subpolitischen Akteure könnten mit dem üblichen Vokabular einer politischen Soziologie strategischer Gruppen bzw. der daran ansetzenden Professionssoziologie beschrieben werden. Es handelt sich jedoch um eine Form »passiver Subpolitik«, die sich selbst nicht in Form politischen Entscheidens beschreiben würde (Holzer/Sørensen 2003). Professionen werden de facto zu subpolitischen Akteuren, wenn »Entscheidungen« über den Einsatz von Technologien ihnen überlassen bleiben. Genau die Zurechungsmöglichkeit einer sich selbst als solche kenntlich machenden Entscheidung wird in diesem Prozess aber in aller Regel eliminiert.

Möchte man Subpolitik als Form von Politik oder gar Herrschaft beschreiben, muss man neben den »klassischen « Einflussquellen positiver und negativer Sanktionen vor allem die Verarbeitung von Ungewissheit, also die »Unsicherheitsabsorption« in Entscheidungsprozessen in Rechnung stellen. Wie wir oben beschrieben haben, ist naturwissenschaftliches Wissen selten eindeutig genug, um eine (einzig richtige) Entscheidung über Risiken zu begründen. Natürlich werden Festlegungen kommuniziert, die Kontroversen abkürzen und Entscheidungsalternativen strukturieren - und in diesem Sinne Unsicherheit absorbieren. Der Schritt zur Konstruktion einer Entscheidung ist aber vor allem auf die Wahrnehmung durch die Politik selbst zurückzuführen: Dieser liegt nichts näher, als ihre Umwelt in der Form von Entscheidungen wahrzunehmen, obwohl diese, zumindest im Fall der Wissenschaft, gar nicht damit befasst ist. Expertenwissen muss in der Regel künstlich »versteift « werden, um politische Entscheidungen zu begründen (Luhmann 2000, S. 161). Die Subpolitik professioneller Experten muss daher nicht als Konkurrent oder gar Substitut »klassischer « staatlicher Politik begriffen werden. Sie ist vielmehr ein notwendiges Korrelat einer Politik, die sich nach wie vor - auch unter der Bedingung unzureichenden oder ungewissen Wissens - dann und wann festlegen muss. Zu einem gewissen Grad kann diese Festlegung unterlaufen werden, indem die Politik einerseits Entscheidungen auf andere und deren Fachwissen zurechnet, andererseits durch den »Streit der Experten« einen höheren Grad an Vorläufigkeit und damit Änderbarkeit eigener Entscheidungen sicherstellt.

10) Man könnte anhand dieses Aspekts die Provokation von Subpolitik näher herausarbeiten, indem man sie mit jener »Herstellung von Sichtbarkeit« vergliche, die für staatliche Politik und die Politik sozialer Bewegungen typisch ist. Vgl. hierzu Nassehi (2002). 
Die Abhängigkeit des »kooperativen Staats« (Voigt 1995) von Professionen oder epistemic communities zeigt sich nicht unbedingt darin, dass diese durch bindende, d.h. Befolgung erwartende Entscheidungen das genuine Feld der Politik übernehmen würden. Zwar werden Entscheidungsprämissen, weniger aber die Entscheidungen selbst, außerhalb der Politik formuliert. Dieser vorgelagerte Bereich der Festlegung von Prämissen und Erwartungen ist nur teilweise politikanalog zu konzipieren. Die Unsicherheitsabsorption findet oft gar nicht in Form autoritativer Entscheidungen statt, sondern in Form sich gegenseitig stützender Normalitätserwartungen. Die autonome Normierung und Standardisierung innerhalb professioneller Gruppen oder ganzer Organisationsfelder vollzieht sich in vielen Bereichen nicht auf der Basis politisch zurechenbarer und sanktionierter Satzungen, sondern in Form scheinbar unverbindlicher Regeln und Standards (Brunsson/Jacobsson 2000). Insofern es sich um eine Form der Institutionalisierung von Erwartungen handelt, die ohne wirksamen Sanktionsmechanismus oder Weberschen Erzwingungsstab auskommt, könnte man diese Prozesse ebenso gut als informale Rechtsentwicklung, analog zum Begriff der Subpolitik also als eine Form des »Subrechts « beschreiben (vgl. hierzu Teubner 1996; 1997). ${ }^{11}$

Im Hinblick auf die Entwicklung regulativer Politik bedeutet die zunehmende Prominenz außerpolitischer Entscheidungsprämissen und Normen erst einmal den Abschied von zu hoch geschraubten Erwartungen an politische Kontroll- und Interventionsmöglichkeiten. Insofern dabei Kompetenzfelder für neue Akteure - vor allem für nichtstaatliche - entstehen, sind diese gerade nicht durch Kontrolle und Vorhersehbarkeit charakterisiert. Man sollte den Fehler vermeiden, in einen oft durch die Vermeidung von Festlegungen geprägten Prozess zu schnell zurechenbare Akteursstrategien hineinzulesen. Der Zuwachs an dem politischen System vorgelagerter Unsicherheitsabsorption geht nicht einseitig auf Kosten des Spielraums staatlich-politischer Entscheidung. Diese bleibt vielmehr in der komfortablen Position, gerade dann, wenn Nichtwissen und Nichtentscheidbarkeit festgestellt werden, zu einer Entscheidung finden zu müssen. In solchen Fällen könnten wir eher von einer »Herrschaft kraft Nichtwissen « als von einer »Herrschaft kraft Ingenieurwissen« sprechen: Die Akzeptanz der politischen Entscheidung gründet sich dann vor allem auf die Nicht-»Entscheidbarkeit « aufgrund vorhandenen Wissens. ${ }^{12}$ Mit anderen Worten: Die Spielräume einer staatlichen Politik der Ungewissheit werden durch Subpolitik nicht beseitigt, sondern gerade geschaffen. Die synchrone und sich wechselseitig irritierende Entscheidungs- und Normierungspraxis in und außerhalb der formalen Politik macht es allerdings schwierig, diesen Pro-

11) Als Folge dieses Prozesses entsteht ein verfassungssystematisches Problem, das wir an dieser Stelle zumindest andeuten möchten: Nicht mehr die alleinige Orientierung an der Zähmung und Bindung des politischen Souveräns kann im Mittelpunkt verfassungsrechtlicher Konstruktionen stehen. Vielmehr muss es um die Entwicklung möglicher Selbstbindungen der Gegenwart gehen, um den »Angriff der Gegenwart auf die Zukunft « - der nunmehr nicht allein durch ungezähmte staatliche Macht möglich ist - verfassungsverträglich zu institutionalisieren. Erforderlich wäre dementsprechend ein Funktionswandel von der Machtbegrenzung zur Zukunftserhaltung durch Selbstbindung. Als Folge dieser Entwicklung werden Verfassungskonzeptionen interessant, in denen die Institution der modernen Verfassung als Institutionalisierung der kollektiven »Selbstbindung « und als mögliches Potential, die Kreativität der Gesellschaft im Umgang mit Zukunftsungewissheit zu erhöhen, verstanden wird. Das gemeinsame Merkmal dieser rechtlichen Regelungsstrukturen liegt in einer Selbstbindung der Gesellschaft, durch die sie das Ziel verfolgt, sich selbst daran zu hindern, in einem zukünftigen Zustand kollektiver Unvernunft die einmal als vernünftig erkannten Grundlagen ihrer Existenz zu zerstören (vgl. Elster 1987; Holmes 1988; Preuß 1994). Kennzeichnend für diesen Typus ist, dass sie sich gerade nicht, wie das für vertragliche oder gesetzliche Bindungen charakteristisch ist, darauf beschränken, Handlungsmöglichkeiten und Gegenseitigkeitsverhältnisse für die Zukunft zu stabilisieren - also Sicherheit für eine ungewisse Zukunft zu erlangen -, sondern sich vielmehr auf die »Sicherung von Sicherheiten« richten und insofern reflexiv sind (Kaufmann 1973, S. 77).

12) Ähnlich das Fazit von Bogner/Menz (2002, S. 398), die daraus aber u.E. etwas zu schnell auf eine Auflösung politischer »Rationalität« schließen. 
zess noch sinnvoll in der Semantik der »Herrschaft« zu beschreiben. Das legt den Schluss nahe, dass die Gesellschaft der Zweiten Moderne nicht mehr als im aristotelischen Sinne aus herrschenden und beherrschten Teilen zusammengesetzte vorstellbar ist. Die Simplifizierung des Zusammenhangs von Macht und Entscheidung und die Zurechnung auf voneinander differenzierte Teile eines Ganzen, die der Herrschaftsbegriff impliziert, helfen nicht weiter. Die theoretische Herausforderung liegt vielmehr gerade darin, sich von der Vorstellung einer souveränen Entscheidungspraxis, die sowohl auf überlegenes Wissen als auch auf Sicherstellung der eigenen Folgen vertrauen kann, zu lösen.

\section{Schluss}

Wir haben in diesem Aufsatz versucht, eine Alternative zu einer unserer Meinung nach zu linearen Interpretation des politischen und rechtlichen Umgangs mit »neuen« Risiken zu entwickeln. Insbesondere wollten wir der Tendenz widersprechen, für ein einseitig auf staatliche Macht fixiertes Modell der regulativen Politik im Rechts- und Wohlfahrtsstaat ein um die überlegene Definitionsmacht von Experten und Professionen kreisendes Herrschaftsmodell zu substituieren. Bei näherem Hinsehen erweist sich das dafür in Anspruch genommene wissenschaftlich-technische Wissen oft als sehr viel uneindeutiger, als es mit derartigen Technokratiethesen vereinbar wäre. Deshalb bildet nicht die faktische Entscheidungskompetenz, sondern der kompetente Umgang mit Nichtwissen und Ungewissheit den Dreh- und Angelpunkt der Veränderungen von Politik und Recht im Prozess reflexiver Modernisierung.

Für das Recht ergibt sich daraus die schwer zu bewältigende Herausforderung, Belastungen der Zukunft durch gegenwärtige Entscheidungen unter Rückgriff auf vorhandenes Wissen zu regulieren. Dabei kann es jedoch allenfalls um die Kanalisierung von Konflikten gehen, kaum um die Vermeidung schädlicher Folgen schlechthin. Für die (staatliche) Politik ist die Lage ambivalenter: Einerseits steht ihr die Möglichkeit offen, gerade die Lücken des professionellen Nichtwissens zum Anlass von Entscheidungen zu nehmen und Ungewissheit so produktiv zu nutzen. Sie muss sich dann aber andererseits auf die Anerkennung von Nichtwissen und auf die daraus resultierende Instabilität von Erwartungen und die Möglichkeit von Enttäuschungen einlassen. Nicht nur eindeutiges Expertenwissen, sondern auch der kompetente Umgang mit Nichtwissen wird vor diesem Hintergrund zu einer Quelle gesellschaftlicher Autorität. Diese ist allerdings deutlich instabiler und amorpher, als es mit einem traditionellen Verständnis politischer Herrschaft vereinbar wäre. Es ist kein Zufall, dass sich der Begriff der Herrschaft nicht im Plural verwenden lässt - während die von uns geschilderten Veränderungen eine faktische Pluralisierung der Grundlagen von Herrschaft anzuzeigen scheinen. Die paradoxe Formel einer »Herrschaft kraft Nichtwissen« ist deshalb zu verstehen als eine Heuristik für das Aufspüren neuer Machtdifferentiale, die quer zu dem klassischen, am Vorbild des modernen Staats entwickelten Herrschaftsmodell verlaufen.

\section{Literatur}

Backes, Otto (1986): Kriminalpolitik ohne Legitimität, in: Kritische Vierteljahresschrift für Gesetzgebung 1, S. 315-342.

Baecker, Dirk (1988): Information und Risiko in der Marktwirtschaft. Frankfurt/Main: Suhrkamp.

Baecker, Dirk (1991): Womit handeln Banken? Eine Untersuchung zur Risikoverarbeitung in der Wirtschaft. Frankfurt/Main: Suhrkamp.

Bauman, Zygmunt (1992): Moderne und Ambivalenz. Das Ende der Eindeutigkeit. Hamburg: Junius.

BCBS (1999): Basel Committee on Banking Supervision: The New Basel Accord (Consultative Paper 1). Basel: Bank for International Settlements.

BCBS (2001): Basel Committee on Banking Supervision: The New Basel Accord (Consultative Paper 2). Basel: Bank for International Settlements. 
BCBS (2003): Basel Committee on Banking Supervision: The New Basel Accord (Consultative Paper 3). Basel: Bank for International Settlements.

Beck, Ulrich (1986): Risikogesellschaft. Auf dem Weg in eine andere Moderne. Frankfurt/Main: Suhrkamp.

Beck, Ulrich (1988): Gegengifte. Die organisierte Unverantwortlichkeit. Frankfurt/Main: Suhrkamp.

Beck, Ulrich (1993): Die Erfindung des Politischen. Zu einer Theorie reflexiver Modernisierung. Frankfurt/Main: Suhrkamp.

Beck, Ulrich (1996): Wissen oder Nicht-Wissen? Zwei Perspektiven »reflexiver Modernisierung «, in: Ulrich Beck/Anthony Giddens/Scott Lash (Hg.), Reflexive Modernisierung. Eine Kontroverse. Frankfurt/ Main: Suhrkamp, S. 289-315.

Beck, Ulrich (1999): Subpolitics: ecology and the disintegration of institutional power, in: World Risk Society. Cambridge: Polity, S. 91-108.

Beck, Ulrich/May, Stefan (2001): Gewusstes Nicht-Wissen und seine rechtlichen und politischen Folgen: Das Beispiel der Humangenetik, in: Ulrich Beck/Wolfgang Bonß (Hg.), Die Modernisierung der Moderne. Frankfurt/Main: Suhrkamp, S. 247-260.

Bogner, Alexander/Menz, Wolfgang (2002): Wissenschaftliche Politikberatung? Der Dissens der Experten und die Autorität der Politik, in: Leviathan 30(3), S. 384-399.

Brunsson, Nils/Jacobsson, Bengt (2000): The contemporary expansion of standardization, in: Nils Brunsson/Bengt Jacobsson (Hg.), A World of Standards. Oxford: Oxford University Press, S. 1-17.

de Swaan, Abram (1993): Der sorgende Staat. Frankfurt/New York. Campus.

Denninger, Erhard (1988): Der Präventions-Staat, in: Kritische Justiz Jg. 21(H. 1), S. 1-15.

Denninger, Erhard (1992): Technologische Rationalität, ethische Verantwortung und postmodernes Recht, in: Kritische Vierteljahresschrift für Gesetzgebung und Rechtswissenschaft, S. 123-139.

Di Fabio, Udo (1993): Grundrechte im präzeptoralen Staat am Beispiel hoheitlicher Informationstätigkeit, in: JZ, S. 689 ff.

Dose, Nicolai (2003): Politisch-administrativer Umgang mit Nichtwissen. München: unveröffentl. Manuskript.

Eatwell, John/Taylor, Lance (2000): Global Finance at Risk: The Case for International Regulation. New York: New Press.

Elster, Jon (1987): Subversion der Rationalität. Frankfurt/New York: Campus.

Ewald, François (1986): L'Etat-Providence. Paris: Édition Grasser \& Fasquell.

Forsthoff, Ernst (1971): Der Staat der Industriegesellschaft. München: C.H. Beck.

Francke, Robert (1994): Ärztliche Berufsfreiheit und Patientenrechte - Eine Untersuchung zu den verfassungsrechtlichen Grundlagen des ärztlichen Berufsrechts und des Patientenschutzes. Stuttgart: Ferdinand Enke Verlag.

Friedrich, Carl J. (1958): Authority, reason, and discretion, in: Carl J. Friedrich (Hg.), Authority (Nomos I). Cambridge, MA: Harvard University Press, S. 28-48.

Fürst, Dietrich (1987): Die Neubelebung der Staatsdiskussion. Veränderte Anforderungen an Regierung und Verwaltung in westlichen Industriegesellschaften, in: Jahrbuch zur Staats- und Verwaltungswissenschaft. Band 1. Baden-Baden: Nomos, S. 261-284.

Gallati, Reto (2003): Risk Management and Capital Adequacy. New York: McGraw-Hill.

Gill, Bernhard/Bizer, Johann/Roller, Gerhard (1998): Riskante Forschung. Zum Umgang mit Ungewissheit am Beispiel der Genforschung in Deutschland. Berlin: edition sigma.

Goeschel, Alexandra (2002): Wissen und Recht. München: unveröffentl. Arbeitspapier.

Grimm, Dieter (1986): Verfassungsrechtliche Anmerkungen zum Thema Prävention, in: Kritische Vierteljahresschrift für Gesetzgebung 69, S. 38-54.

Grimm, Dieter (1991): Wandel der Staatsaufgaben und die Krise des Rechtsstaats, in: Die Zukunft der Verfassung. Frankfurt/Main: Suhrkamp, S. 159-176. 
Habermas, Jürgen (1985): Die neue Unübersichtlichkeit. Frankfurt/Main: Suhrkamp.

Habermas, Jürgen (1992): Faktizität und Geltung. Beiträge zur Diskurstheorie des Rechts und des demokratischen Rechtsstaats. Frankfurt/Main: Suhrkamp.

Hesse, Hans Albrecht (1993): Der Schutzstaat geht um, in: JZ, S. 741-748.

Hesse, Hans Albrecht (1994): Der Schutzstaat. Rechtssoziologische Skizzen in dunkler Zeit. BadenBaden: Nomos.

Hiller, Petra (1993): Der Zeitkonflikt in der Risikogesellschaft: Risiko und Zeitorientierung in rechtsförmigen Verwaltungsentscheidungen. Berlin: Duncker \& Humblot.

Hirsch, Joachim (1980): Der Sicherheitsstaat. Frankfurt/Main: EVA.

Holmes, Stephen (1988): Precommitment and the paradox of democracy, in: Jon Elster/Rune Slagstadt (Hg.), Constitutionalism and Democracy. Cambridge: Cambridge University Press, S. 195-240.

Holzer, Boris/Beck, Ulrich (2004): Global Financial Risks and Transnational Regime-Building: Basel II and the German Domestic Policy Discourse (Research Report to the World Society Foundation, Zurich). München: Institut für Soziologie der LMU München.

Holzer, Boris/Millo, Yuval (2005): From Risks to Second-order Dangers in Financial Markets: Unintended Consequences of Risk Management Systems, in: New Political Economy 10 (forthcoming).

Holzer, Boris/Sørensen, Mads P. (2003): Rethinking subpolitics: Beyond the >iron cage < of modern politics? in: Theory, Culture \& Society 20(2), S. 79-102.

Kaufmann, Franz-Xaver (1973): Sicherheit als soziologisches und sozialpolitisches Problem. Untersuchung zu einer Wertidee hochdifferenzierter Gesellschaften. Stuttgart: Enke.

Ladeur, Karl-Heinz (1995): Das Umweltrecht der Wissensgesellschaft. Berlin: Duncker \& Humblot.

Ladeur, Karl-Heinz (1999): Risikobewältigung durch Flexibilisierung und Prozeduralisierung des Rechts - Rechtliche Bindung von Ungewissheit oder Selbstverunsicherung des Rechts? in: Alfons Bora (Hg.), Rechtliches Risikomanagment. Form, Funktion und Leistungsfähigkeit des Rechts in der Risikogesellschaft. Berlin: Duncker \& Humblot, S. 41-64.

Luhmann, Niklas (1972): Rechtssoziologie (2 Bde.). Reinbek b. Hamburg: Rowohlt.

Luhmann, Niklas (1988): Macht. Stuttgart: Enke.

Luhmann, Niklas (1989): Zwei Seiten des Rechtsstaates, in: The Institute of Comparative Law in Japan (Hg.), Conflict and Integration: Comparative Law in the World Today. The 40th Anniversary of the Institute of Comparative Law in Japan, Chuo University, 1988. Tokyo: Chuo University Press, S. 493-506.

Luhmann, Niklas (1990): Risiko und Gefahr, in: Soziologische Aufklärung 5. Opladen: Westdeutscher Verlag, S. 131-169.

Luhmann, Niklas (1991): Soziologie des Risikos. Berlin/New York: Walter de Gruyter.

Luhmann, Niklas (1992): Ökologie des Nichtwissens, in: Beobachtungen der Moderne. Opladen: Westdeutscher Verlag, S. 149-220.

Luhmann, Niklas (1993): Das Recht der Gesellschaft. Frankfurt/Main: Suhrkamp.

Luhmann, Niklas (1994): Der »Radikale Konstruktivismus« als Theorie der Massenmedien? Bemerkungen zu einer irreführenden Debatte, in: Communicatio Socialis 27(1), S. 7-12.

Luhmann, Niklas (2000): Die Politik der Gesellschaft. Frankfurt/Main: Suhrkamp.

Lütz, Susanne (1999): Zwischen »Regime« und »kooperativem Staat«. Bankenregulierung im internationalen Mehr-Ebenen-System, in: Zeitschrift für Internationale Beziehungen 6(1), S. 9-40.

Lyotard, Jean-Francois (1986): Das postmoderne Wissen. Ein Bericht. Graz/Wien: Passagen.

March, James G./Simon, Herbert A. (1958): Organizations. New York/Chichester: John Wiley \& Sons.

Marquard, Odo (1981): Inkompetenzkompensationskompetenz? Über Kompetenz und Inkompetenz der Philosophie, in: Abschied vom Prinzipiellen. Stuttgart: Reclam, S. 23-38.

May, Stefan (2002): Institutionelle Entgrenzungen durch Nichtwissen. München: Dissertation, LMU München. 
May, Stefan (2004): Rechtspolitische Nebenfolgen und Entscheidungskonflikte in der Biomedizin, in: Ulrich Beck/Christoph Lau (Hg.), Entgrenzung und Entscheidung. Frankfurt/Main: Suhrkamp, S. 193-208.

May, Stefan (2005): Reflexiver Konstitutionalismus? Analysen zum Rechtsformenwandel im Prozess reflexiver Modernisierung. Antragspapier zur dritten Forschungsphase. München: Sonderforschungsbereich 536 .

May, Stefan (2005a, i.E.): Neue Risiken, neue Regeln. Frankfurt/New York: Campus.

May, Stefan/Holzinger, Markus (2003): Autonomiekonflikte der Humangenetik. Professionssoziologische und professionsrechtliche Aspekte einer Theorie reflexiver Modernisierung. Opladen: Leske + Budrich.

May, Stefan/Böschen, Stefan (2005, i.E.): Politik im Niemandsland. Wiesbaden: VS.

Murck, Manfred (1980): Soziologie der öffentlichen Sicherheit. Frankfurt/New York: Campus.

Nassehi, Armin (2002): Politik des Staates oder Politik der Gesellschaft? Kollektivität als Problemformel des Politischen, in: Kai-Uwe Hellmann/Rainer Schmalz-Bruns (Hg.), Theorie der Politik. Niklas Luhmanns politische Soziologie. Frankfurt/Main: Suhrkamp, S. 38-59.

Parsons, Talcott (1939): The professions and social structure, in: Social Forces 17(4), S. 457-467.

Perrow, Charles (1984): Normal Accidents: Living with High-Risk Technologies. New York: Basic Books.

Peters, Bernhard (1993): Die Integration moderner Gesellschaften. Frankfurt/Main: Suhrkamp.

Preuß, Ulrich K. (1994): Revolution, Fortschritt und Verfassung. Zu einem neuen Verfassungsverständnis. Frankfurt/Main: Fischer.

Preuß, Ulrich K. (1996): Risikovorsorge als Staatsaufgabe, in: Dieter Grimm (Hg.), Staatsaufgaben. Frankfurt/Main: Suhrkamp, S. 523-553.

Ravetz, Jerome R. (1987): Usable knowledge, usable ignorance, in: Knowledge 9(1), S. 87-116.

Rorty, Richard (1988): Contingency, Irony, Solidarity. Cambridge: Cambridge University Press.

Schelsky, Helmut (1965): Der Mensch in der wissenschaftlichen Zivilisation (1961), in: Auf der Suche nach Wirklichkeit. Düsseldorf/Köln: Diederichs, S. 439-480.

Scherzberg, Arno (2002): Wissen, Nichtwissen und Ungewissheit im Recht, in: C. Engel/Jost Halfmann (Hg.), Wissen - Nichtwissen - Unsicheres Wissen. Baden-Baden: Nomos, S. 113-145.

Schmidtke, Jörg (1995): »Nur der Irrtum ist das Leben, und das Wissen ist der Tod.« Das Dilemma der Prädiktiven Genetik, in: Beck-Gernsheim (Hg.), Welche Gesundheit wollen wir? Dilemmata des medizinischen Fortschritts. Frankfurt/Main: Suhrkamp, S.25-32.

Schroeder-Kurth, Traute M. (1994): Rechtliche Apekte genetischer Diagnostik. Alles was Recht ist? in: Medizinische Genetik 6, S. 396-399.

Stehr, Nico (1994): Arbeit, Eigentum und Wissen. Zur Theorie von Wissensgesellschaften. Frankfurt/ Main: Suhrkamp.

Steinbicker, Jochen (2001): Zur Theorie der Informationsgesellschaft. Opladen: Leske + Budrich.

Stichweh, Rudolf (1994): Wissenschaft, Universität, Professionen. Soziologische Analysen. Frankfurt/ Main: Suhrkamp.

Strulik, Torsten (2000): Risikomanagement globaler Finanzmärkte. Frankfurt/New York: Campus.

Teubner, Gunther (1989): Recht als autopoietisches System. Frankfurt/Main: Suhrkamp.

Teubner, Gunther (1996): Des Königs viele Leiber. Die Selbstdekonstruktion der Hierarchie des Rechts, in: Soziale Systeme 2(2), S. 229-255.

Teubner, Gunther (1997): 'Global Bukowina': legal pluralism in the world society, in: Global Law Without a State. Aldershot: Dartmouth, S. 3-28.

Ullrich, Otto (1979): Technik und Herrschaft. Frankfurt/Main: Suhrkamp.

Voigt, Rüdiger (1995): Der kooperative Staat: Auf der Suche nach einem neuen Steuerungsmodus, in: Rüdiger Voigt (Hg.), Der Kooperative Staat. Krisenbewältigung durch Verhandlung? Baden-Baden: Nomos, S. 33-92. 
Weber, Max (1980): Wirtschaft und Gesellschaft. Grundriss der verstehenden Soziologie (orig. 19211922). Tübingen: J. C. B. Mohr (Paul Siebeck).

Wehling, Peter (2001): Jenseits des Wissens? Wissenschaftliches Nichtwissen aus soziologischer Perspektive, in: Zeitschrift für Soziologie 30(6), S. 465-484.

Willke, Helmut (1992): Ironie des Staates. Grundlinien einer Staatstheorie polyzentrischer Gesellschaft. Frankfurt/Main: Suhrkamp.

Wolf, Rainer (1987): Zur Antiquiertheit des Rechts in der Risikogesellschaft, in: Leviathan 15(3), S. 357391.

Wolf, Rainer (1988): »Herrschaft kraft Wissen « in der Risikogesellschaft, in: Soziale Welt 39, S. 164-187.

Wrong, Dennis H. (1995): Power: Its Forms, Bases and Uses. Brunswick, NJ: Transaction.

Zapf, Wolfgang/Breuer, Sigrid/Hampel, Jürgen/Krause, Peter/Mohr, Hans-Michael/Weick, Stefan/Wiegand, Erich (1987): Individualisierung und Sicherheit. Untersuchungen zur Lebensqualität in der Bundesrepublik Deutschland. München: C.H. Beck.

Boris Holzer Ph.D.

LMU München, Institut für Soziologie, Konradstr. 6, 80801 München B.Holzer@Lmu.de

Dr. Stefan May, SFB 536, LMU München, Theresienstr. 37, 80333 München Stefan.May@soziologie.uni-muenchen.de 